\title{
Die Ethnologie an den Schweizer Universitäten
}

\section{Eine junge Disziplin: Ihr Selbstverständnis heute}

Wolfgang Rudolph schrieb vor einigen Jahren: «An verhätschelten Theorien hat es der Ethnologie im Laufe ihrer Entwicklung nicht gefehlt, ob sie nun unter «Geschichte», "Soziologie» oder einem andern Etikett angeboten wurden. Vielleicht sollte man einmal versuchen, die Ethnologie unter «Ethnologie» anzubieten. 1) Man gibt heute vor allem im englischen, $z$. T. auch im französischen Sprachbereich der Bezeichnung "Anthropologie» oder, wenn man sie je nach dem thematischen Akzent näher etikettieren will, «Kultur-» oder «Sozialanthropologie» den Vorzug.

Es kann in dieser kurzen Präsentation weder um eine wissenschaftssystematische Einordnung noch um eine methodologische Einführung in die verschiedenen Richtungen der Völkerkunde gehen, sondern nur um eine knappe Übersicht über Gegenstand, Arbeitsweisen und fachspezifische Theoriebildung, wie sie heute im Vordergrund stehen. Das Schema ${ }^{2}$ möchte weniger die thematische Breite und geographische Weite der Disziplin zum Ausdruck bringen - beides erhellt aus den nachfolgend kurz skizzierten Curricula und Spezialisierungen der Universitäts-Institute - als vielmehr ihre verschiedenen theoretischen und praxisbezogenen Ansätze aufzeigen.

Der Ausgangspunkt unserer wissenschaftlichen Arbeit ist entsprechend entweder primär akademisch im Sinn einer auf dem Gebiet der Ethnohistorie, der Kulturhistorie, der Sozialanthropologie, der Kulturanthropologie, der strukturalen Theorie oder auch einer interdisziplinären Betrachtungsweise formulierten Hypothese mit dem Ziel ihrer Prüfung - oder einfach die Zielvorstellung eines systematischen Beitrags zu neuen Kenntnissen in einer der genannten Richtungen. Dabei ist zu beachten, daß diese sich gegenseitig weder auszuschließen brauchen noch klar abgrenzbar sind. Der Ausgangspunkt kann aber auch ein mehr auf die Praxis ausgerichteter Auftrag von seiten einer Institution oder auch ein selbstgewählter Dienst an Volk und Kultur sein. Das dispensiert natürlich nicht von wissenschaftlicher Objektivität und methodischer Exaktheit.

$\mathrm{Da}$ die soziokulturelle Situation im «Feld" in ihrer räumlich-zeitlichen Dimension und intrakulturellen Variabilität, in ihrer ökologischen Bedingtheit und historischen Einmaligkeit unser Basis-Laboratorium ist, wird niemand bestreiten. Auch eine reine Schreibtisch-Arbeit, eine Analyse oder ein Vergleich aufgrund von Museumsobjekten, offiziellen Dokumenten oder audiovisuellen Zeugnissen oder aufgrund bereits publizierter Werke anderer gründet schließlich auf direkter ethnographischer Forschung. Man hat in den letzten 20 Jahren versucht, diese Datenerhebung im Feld authentischer, wissenschaftlicher durchzuführen. Entsprechend hat man denn auch dafür, in Anlehnung an die exakten Methoden der Naturwissenschaften, den Titel «Ethnoscience» geprägt. Die Sache an sich ist nicht neu. Jeder gute Ethnograph war schon früher bemüht, die konkrete ethnographische Wirklichkeit nicht aus seiner eigenen europa-zentrischen Weltanschauung und Perspektive heraus und nicht in seiner eigenen kulturgebundenen und darum weithin pseudowissenschaftlichen Terminologie zu erfassen, sondern so wie die Kultur eines Volkes sich selbst darstellt. Die neue Betonung dieser «emic»-Situation des Feldforschers und die Forderung ihrer Übersetzung in eine zwar präzise, d. h. nicht entleerte aber auch nicht parochiale, zugleich aber allgemein verständliche wissenschaftliche Darstellung (etic) hat sich in den stark problem-betonten Arbeiten der heutigen Ethnologie positiv ausgewirkt.

Es ist seit Claude Lévi-Strauss in manchen Kreisen unserer Wissenschaft Mode geworden, das ethnographische Material und das kulturelle Phänomen als Ganzes nach formal-strukturalen Modellen zu analysieren, um aus der Dialektik seiner Schöpfungen und seiner denkerisch-spielerischen Ausdrucksformen die menschliche Geistigkeit selbst neu zu überdenken. Andere bleiben lieber auf der Ebene der leichter verifizierbaren soziokulturellen Fakten. Auf der Basis ihrer gesammelten Daten gelangen sie stufenweise von der einfachen Deskription zur assoziativen, zur funktionalen, zur systembegrenzenden Analyse und zur mehr oder weniger gültigen globalen Erfassung. Durch quantitative und qualitative vergleichende Auswertung und durch Zusammenarbeit mit den Nachbardisziplinen erstreben sie schließlich die Prüfung bestehender und die Formulierung neuer Hypothesen im Bereich sozialer und kultureller Adaptation und Integration und der kulturellen Dynamik im weitesten Sinn. Andern wiederum geht es primär um funktionelle Querverbindungen und Gesetzlichkeiten oder um kulturhistorische Zusammenhänge. $\mathrm{Da} \beta$ in dieser komparativen

Prof. Dr. Hugo Huber, Ethnologisches Seminar der Universität Freiburg, Institut Froideville, 1725 Posieux 


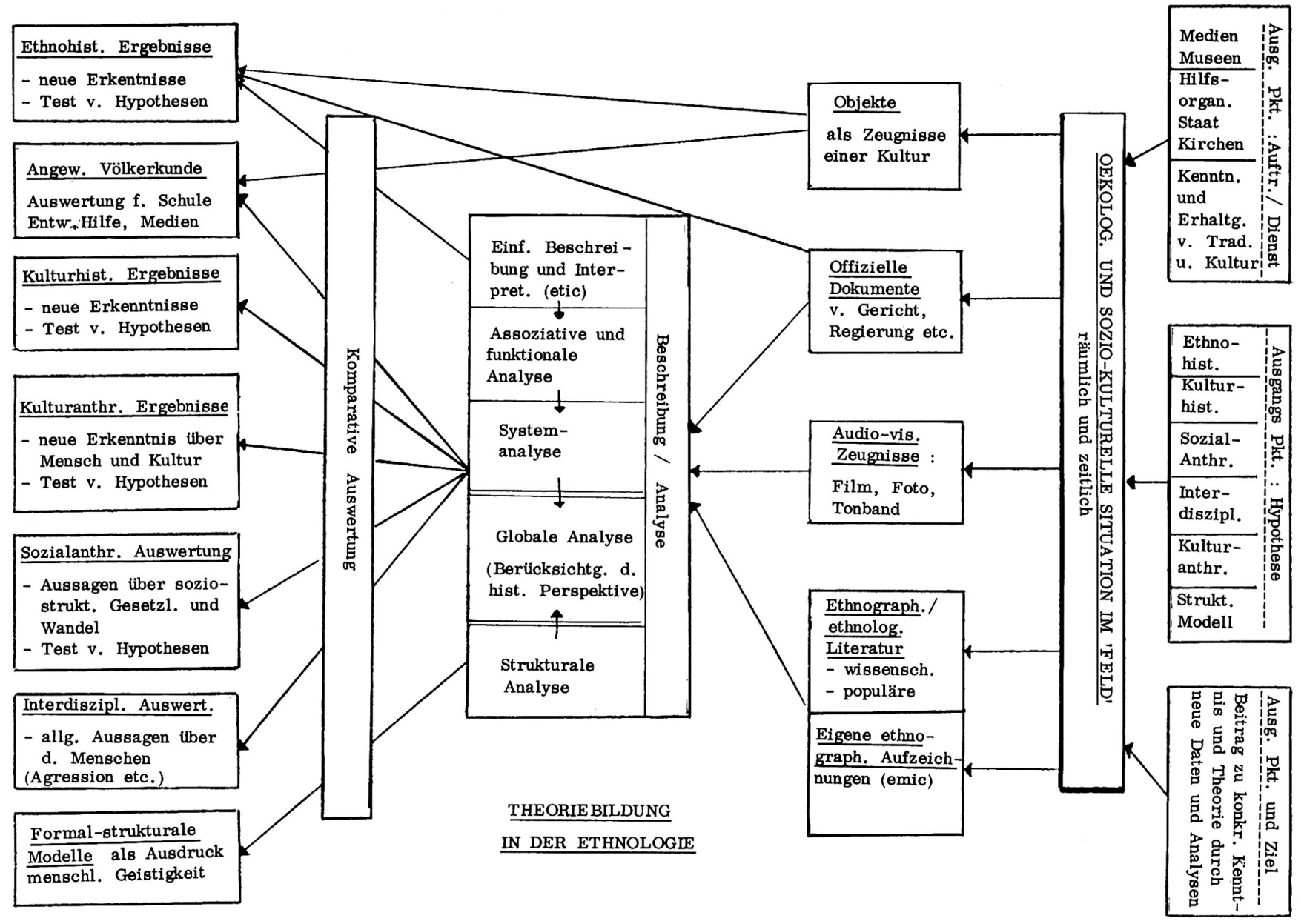


Auswertung, methodisch gesehen, die Fragen der Meßbarkeit und der Komparabilität, vor allem die Definierung und Isolierung gültiger Vergleichskategorien, Probleme aufwerfen, liegt auf der Hand. Gerade im interkulturellen Vergleich sind ja auch für den Fachethnologen kognitive Schranken und Übersetzungsschwierigkeiten zu erwarten.

Man spürt neuerdings auch in der Schweiz ein vermehrtes Interesse an der Ethnologie, nicht zuletzt deshalb, weil sie versucht, in Zusammenarbeit mit andern human-wissenschaftlichen Disziplinen gesamtmenschliche Probleme anzugehen wie etwa Dürre und Hunger in der Welt, die Stellung der Frau und der Alten, internationaler Tourismus, wirtschaftliche Kooperation und Selbsthilfe und soziale Befreiung in Entwicklungsgebieten und die Zukunft ethnischer Minderheiten. Wenn auch heute noch weitgehend die sogenannten schriftlosen Kulturen im Mittelpunkt ihrer Arbeiten stehen, so geht es dabei nicht mehr um eine grundsätzliche Einschränkung. Mit andern Worten, die Ethnologie wird mehr und mehr zur allgemeinen Anthropologie, zur engagierten Humanwissenschaft.

\section{Unterricht und Forschung an den Hochschulen}

Die nachfolgende Zusammenstellung stützt sich im wesentlichen auf die Selbstdarstellung der ethnologischen Institute und Seminare der Schweiz ${ }^{3)}$ aufgrund einer schriftlichen Befragung, sowie auf ihre Unterrichts- und Forschungsprogramme, wie sie laufend im Bulletin der Schweizerischen Ethnologischen Gesellschaft publiziert werden. Da die von den Völkerkunde-Museen durchgeführten Forschungen unter eigener Rubrik erscheinen, werden sie hier nicht eigens erwähnt.

\section{BASEL}

Charakteristisch für die Ethnologie an der Universität Basel ist ihre enge historische Bindung an das Völkerkunde-Museum, das im Kern auf das Jahr 1849 zurückgeht. Die international bekannten Forscher der Jahrhundertwende, Fritz und Paul Sarasin, arbeiteten noch als Privatgelehrte. Der stufenweise Einbau in die akademische Lehre begann 1914 mit der Habilitation und dem Lehrauftrag von Felix Speiser. 1917 wurde daraus das Extraordinariat und 1963/64 der eigentliche Lehrstuhl mit dem Ordinariat unter Alfred Bühler. Gleichzeitig ist die Personalunion von Museumsleitung und akademischem Lehramt, nicht aber die enge Zusammenarbeit auf dem Gebiet der Lehre und Forschung (gemeinsame Felduntersuchungen, Museum als «Laboratorium» für die Ethnologiestudenten) aufgegeben worden. Seit 1970 ist Meinhard Schuster Inhaber des Lehrstuhls und Leiter des Ethnologischen Seminars. Man versucht trotz Knappheit an Dozenten durch parallele Vorlesungs- und Semiarzyklen ein möglichst umfassendes Bild von der Ethnologie (regional, ver- gleichend-theoretisch, akkulturations-ethnologisch, ethnotechnologisch) zu vermitteln. Der früher überwiegend kulturhistorisch-vergleichende Ansatz ist zugunsten einer intensiveren Lokalforschung unter Berücksichtigung gesamtkultureller Zusammenhänge zurückgetreten. Thematisch stehen neben den ergologischen und technologisch-ökonomischen Forschungen im Bereich der Textilien und der Keramik jene auf den Gebieten der Religions-, Musik- und Kunstethnologie und Fragen zur Sozialorganisation (Klangliederung, Rolle der Frau) im Vordergrund. Die vor allem durch die enge Zusammenarbeit mit dem $\mathrm{Mu}$ seum inspirierte Konzentration auf Papua New Guinea und auf bestimmte Regionen Indonesiens ist in den letzten Jahren durch Einschluß von Ostperu, Kolumbien, Indien und Westafrika bedeutend erweitert worden.

$\mathrm{Zu}$ erwähnen ist die Befruchtung der Ethnologie durch die Zusammenarbeit mit den Nachbardisziplinen, so mit der Geographie im Rahmen der 1923 gegründeter Geographisch-Ethnologischen Gesellschaft, mit der Volkskunde durch die gemeinsamen Übungen in der ethnographischen Feldarbeit, mit der Musikwissenschaft durch die musik-ethnologischen Feldforschungen auf Bali und im hinterindischen Raum - und allgemein auch mit der Ur- und Frühgeschichte. 1964 begann die Herausgabe der Pubilkationsreihe «Basler Beiträge zur Ethnologie».

\section{BERN}

Das Seminar für Ethnologie an der Universität Bern geht auf das Jahr 1966 zurück. Walter Dostal wurde gleichzeitig als erster Inhaber des Lehrstuhls berufen. Regionaler Schwerpunkt war damals der Vordere Orient. Es wurden denn auch entsprechende Untersuchungen über die Dorfkulturen in Südarabien und Anatolien durchgeführt und in enger Zusammenarbeit mit den Ethnologen am Bernischen Historischen Museum mehrere Ausstellungen über die Kulturen dieser Gebiete durchgeführt. In weiteren Feldforschungen ging es um interethnische Beziehungen im Raum Afghanistan, um Akkulturationsprobleme einer Eskimogruppe und um ethnohistorische Untersuchungen im südlichen Tanzania.

Seit 1976 betreut Wolfgang Marschall Lehrstuhl und Seminar. Das hat eine regionale Schwerpunkt-Verlagerung auf Indonesien mit sich gebracht mit einem entsprechenden Angebot auf dem Gebiet von Kultur und Sprache. Im Lehrangebot sind als Ergänzung Vorlesungen über Ostafrika und Kurse in Swahili durch Rupert R. Moser, ferner Spezialvorlesungen über Afghanistan und Zentralasien durch Pierre Centlivres regelmäßig vorgesehen.

1978 hat die neue Publikationsreihe "Studia Ethnologica» die früheren «Veröffentlichungen des Seminars für Ethnologie der Universität Bern» abgelöst. 


\section{FREIBURG}

Der Ethnologieunterricht an der Universität Freiburg begann im Wintersemester 1939/40 mit dem Lehrauftrag von Wilhelm Schmidt, welcher 1938 mit dem von ihm gegründeten Anthropos-Institut nach Posieux umgesiedelt war. 1942 wurde er Ordinarius. Lehrstuhl und Seminar wurden in der Folge nacheinander durch Georg Hölker, Joseph Henninger und Rudolf Rahmann betreut. Gegenwärtiger Inhaber bzw. Leiter (seit 1960) ist Hugo Huber.

Das auf 8 Semester sich erstreckende Lehrprogramm umfaßt heute:

- einen theoretisch-thematischen Zyklus mit einer allgemeinen theoretischen Einführung in die verschiedenen Methoden und Ausrichtungen und in die vordergründigen Themenbereiche der Ethnologie

- einen regionalen Zyklus mit Indien, Schwarzafrika, Ozeanien, Indonesien, Madagaskar und die Indianerkulturen Nordamerikas

- einen Zyklus ausgewählterThemen der afrikanischen Völkerkunde, entsprechend der Spezialisierung des derzeitigen Lehrstuhlinhabers.

In den Einzel- und Gruppenarbeiten im Rahmen des Seminars stehen neben der Theorie des wissenschaftlichen Arbeitens vorab Gegenwartsprobleme in der Dritten Welt zur Diskussion.

Die in den vergangenen 15 Jahren durchgeführten Forschungen des Lehrstuhlinhabers, sowie verschiedener Doktoranden und Lizeanden bezogen sich vornehmlich auf sozialanthropologische und religionsethnologische Fragen in Gebieten von Tanzania, Benin, Kamerun, Taiwan, Bangla Desh und Mexiko. Gegenwärtig steht themamäßig das Alter im Vordergrund. 1972 wurde die Schriftenreihe «Studia Ethnographica Friburgensia» begonnen.

\section{GENÈVE}

L'Université de Genève offre un cours d'ethnologie, une heure par semaine, dans le cadre de la Faculté des Sciences Economiques et Sociales par Marc Sauter. Il est réparti sur deux ans et couvre une introduction abrégée et générale aux principales théories et sujèts thématiques de la discipline.

Lié à l'Université est un centre d'études interdisciplinaires avancées qui depuis 1977 s'appelle «Institut Universitaire d'Etudes du Développement». Dirigé aujourd'hui par A. R. Preiswerk, il offre un centre d'accueil et un lieu de recherches et de réflexion pour les étudiants intéressés aux changements sociaux, économiques et politiques du Tiers-monde. Parmi les quelques trente enseignants il y a d'ethnologues professionnels comme Fabricio Sabelli et Gérald Berthoud.

\section{LAUSANNE}

A l'Université de Lausanne il y a à la Faculté des Sciences Sociales et Politiques un cours d'introduction d'anthropologie culturelle et sociale au niveau propédeutique ( 2 heures hébdomadaires) et un enseignement plus poussé des théories anthropologiques en vue d'une licence en sociologie, en sciences politiques ou en sciences sociales et pédagogiques, les deux donnés par Gérald Berthoud.

\section{LUZERN}

Die Theologische Fakultät hat seit einigen Jahren eine Dozentur für Religionswissenschaft und Ethnologie. Otto Bischofberger, der selbst in Tanzania und Taiwan religionsethnologische und ethnosoziologische Forschungen durchgeführt und sich auch eingehend mit neoafrikanischer Literatur und prophetischen Bewegungen beschäftigt hat, betreut sie.

\section{NEUCHÂTEL}

Depuis 1949 Neuchâtel a un enseignement regulier en ethnologie lié jusqu'à 1974 à la géographie humaine. Jean Gabus, connu par ses nombreuses missions ethnographiques au Sahara et au Cameroun et par ses expositions au Musée d'Ethnographie, fût le premier directeur de l'Institut d'Ethnologie. 1974 l'ethnologie devient branche principale de la licence ès lettres avec Pierre Centlivres comme nouveau directeur. Sa spécialité régionale est l'Asie centrale et le Moyen Orient. L'enseignement est désormais assuré par un professeur ordinaire, deux chargés de cours et un chef de travaux. De plus, un cours d'ethno-musicologie peut être choisi comme branche complémentaire.

Le programme général porte surtout sur l'histoire de la pensée ethnologique à travers des mouvements et des écoles tels que l'évolutionisme, le diffusionisme, le fontionalisme, le structuralisme, les approches de l'anthropologie cognitive et de l'écologie culturelle. Il comprend la méthodologie, l'ethnotechnologie, l'anthropologie économique et sociale. Dans l'ethnologie régionale les Touaregs et les pasteurs nomades de l'Asie et, récemment, les sociétés rurales de la Suisse romande sont devenus un terrain privilégié pour les recherches.

Des exercices pratiques des dernières années ont porté sur la technologie paysanne, sur l'alimentation et l'identité régionale. Des enquêtes plus poussées ont été menées sur l'anthropologie économique des Bongo du Soudan, sur les collectivités rurales jurassiennes, sur la sédentarisation des éleveurs en Nord Afghan, sur les Indiens Wayana-Aparai du Brésil et sur l'évolution du carnaval en Valais.

On espère la réalisation d'un centre de recherche prévu de longue date.

Avec les "Cahiers de l'Institut d'Ethnologie de l'Université de Neuchâtel» l'Institut a depuis 1978 sa publication périodique. 


\section{ZÜRICH}

Alfred Steinmann, langjähriger Direktor der völkerkundlichen Sammlung der Universität Zürich, begann 1939 mit ethnologischen Vorlesungen. Durch seinen langjährigen Aufenthalt in Indonesien hatte er sich in die Kunst und Kultur dieser Inselwelt hineingelebt. Dies blieb, zusammen mit dem südostasiatischen Festland, sein Spezialgebiet. Er habilitierte sich 1941 und wurde 1948 Titularprofessor. Seine Funktion am Museum und an der Universität übernahm später Karl H. Henking, Spezialist auf dem Gebiet der Kunstund Religionsethnologie.

Unabhängig vom Museum entstand 1971 der Lehrstuhl für Ethnologie mit dem Ethnologischen Seminar unter Leitung von Lorenz G. Löffler. Mit seinen derzeitigen vollamtlichen Mitarbeitern Wolfgang Laade, HansPeter Müller, Hanna Rauber, Regula Egli betreut er ein breites Spektrum der völkerkundlichen Wissenschaft. Als Sachgebiete treten vor allem die folgenden hervor: Sozial-, Wirtschafts- und Entwicklungsethnologie, ferner Ethnolinguistik, Ethnomedizin, Ethnopädagogik, Ethnopsychologie, Musikethnologie und Cross-Cultural Analysis. Regionale Spezialisierungen beziehen sich vor allem auf Bangla Desh, Nepal und Westafrika.

Besonderes Gewicht wird auf Forschungspraktika für Studenten gelegt: Bisher hat die Mehrheit der Studenten vor dem Abschluß eine zirka halbjährige Feldforschung im außereuropäischen Raum durchgeführt nach ein- bis zweisemestriger Intensiveinführung in die jeweilige Landes- bzw. Stammessprache. Neben aktuellen Problemen der Minoritäten in Bangla Desh standen sozioökonomische Fragen in Südbenin, Entwicklungsprobleme in Kamerun, sozialanthropologische Forschungen in Westnepal, Fragen der Landreform in Iran, sozialpolitische und rechtliche Belange in Westsumatra, musik-ethnologische Erkundigungen in Sri Lanka und weitere Forschungen in Mexiko, Algerien und Burundi auf dem Programm.

\section{Kommunikation und Zusammenarbeit: Ethnologie und Geographie}

Wie steht es mit der immer wieder betonten Kommunikation und Zusammenarbeit auf universitäter Ebene im Sektor Ethnologie? Man hat von einer Koordination im Lehrangebot, von programmiertem Studienplatzwechsel, von reisenden Dozenten, von interlokalen Seminaren und vom Austausch der Lehrinhalte gesprochen (Bull. SEG 1975/1, S. 1). Wenn auch diese und ähnlich weitreichende Ideen und Vorschläge erwartungsgemäß nicht verwirklicht worden sind, so hat die Gründung der Schweizerischen Ethnologischen Gesellschaft (SEG/SSE) im Juni 1971 doch Erfreuliches in dieser Richtung gebracht. Die Tatsache, daß von ihren über 300 Migliedern mehr als die Hälfte Studenten sind, weist auf ihre starke Verankerung an den Universitäten hin.
Das seit 1972 jährlich zweimal erscheinende Informations-Bulletin bringt neben den Protokollen der Mitgliederversammlungen und den Kommissionsberichten regelmäßig Nachrichten über Lehrveranstaltungen, Forschungsprogramme und Publikationen der einzelnen Institute und Seminarien. Die Arbeit in den verschiedenen Kommissionen (Wissenschaftliche Kommission, Museumskommission, Redaktionskommission, Kommission Dritte Welt, Kommission für audiovisuelle Dokumentation und Kommission für Öffentlichkeitsarbeit) hat nicht nur Lehrstuhlinhaber unter sich näher zusammengebracht, sondern auch die studentischen Vertreter der verschiedenen Universitäten und die Ethnologen der Praxis. Das gleiche trifft für die Jahresversammlungen und Blockseminare zu, welche von ihrer Thematik her gewöhnlich auch die Zusammenarbeit mit den Nachbardisziplinen angeregt haben. Man denkt hierbei vor allem an die wissenschaftlichen Sitzungen und Intensivseminare «Ethnologie und Dritte Welt» (1972), «Ethnologie und Schule» (1974), «Europäische Ethnologie» (1974), «Ethnolinguistik» (1976) und "Agrarprobleme in agronomischer und ethnologischer Sicht» (1978). Daraus erhellt das Interesse der Schweizer Ethnologen für das interdisziplinäre Gespräch. $\mathrm{Ob}$ es in näherer Zukunft zu vermehrter Kooperation mit unseren Nachbardisziplinen kommen wird, hängt von beiden Seiten ab. Ein intensiverer gegenseitiger Kontakt wird neuerdings auch unter den studentischen Gruppen der verschiedenen Universitäten angestrebt. Das Tagungsthema 1976 über die Probleme der Feldforschung aus der Sicht der jungen Ethnologen wies in gleiche Richtung (Vgl. SEG Sondernummer 1976).

Zum Schluß sei noch die Frage nach den Berührungspunkten und nach den Möglichkeiten der Zusammenarbeit zwischen der Ethnologie und der Geographie im besonderen gestellt. Die Einladung zur Gestaltung dieser Sondernummer darf als erfreulicher Schritt in dieser Richtung von seiten der Geographie gewertet werden. A. Buri hat seinerzeit in seinem kurzen Grundsatzreferat zur Jahrestagung der Schweizerischen Ethnologischen Gesellschaft von 1974 über «Ethnologie im Geographieunterricht» (Vgl. Bull. SEG 1974/2, S. 3 ff.) von der «Landschaft als Lebensraum des Menschen" gesprochen und vom "Menschen, der immer mehr ins Zentrum der Schulgeographie rückt». Hier liegt ohne Zweifel der Ansatz zur Begegnung und gegenseitigen Befruchtung unserer beiden Wissenschaften. Wenn auch der Mensch als vernunftbegabtes und schöpferisch-aktives Wesen, vor allem in den Industrieländern, durch seine Technik und sein Wissen weithin über den Zwängen der Natur und der Umwelt steht, so sind doch auch in der Gegenwart noch die ökologischen Bedingungen, die klimatischtopographischen Verhältnisse vor allem für die primitiveren Völker von enormer Bedeutung. Die SahelKatastrophe hat uns dies in den vergangenen Jahren mit erschreckender Deutlichkeit nahegebracht. Beide Disziplinen, Geographie und Ethnologie, mühen sich 
um die Klärung des Zusammenspiels Natur/Mensch/ Kultur. Beide wissen, daß die physisch-geographische Umwelt in manchen Gebieten der Erde auch heute noch die unmittelbare Grundlage menschlicher Existenz und Lebensmeisterung bedeutet. Beide wissen um die Zusammenhänge zwischen Klima, Wirtschaftsform, Siedlungsgeschichte, Demographie, Art derTechniken, Industrien und Kommunikationsmittel und um deren Einfluß auf die sozialen und territorialen menschlichen Gruppierungen und gesellschaftlichen Strukturen und damit auch auf Politik und Ideologien. Die naturgegebene Umwelt hat den Menschen im Lauf der Geschichte zu immer neuen Erfindungen herausgefordert.

Wo steht unsere tatsächliche Zusammenarbeit? A. Buri (a.a.O.) fordert für die Geographielehrer entsprechende Grund- und Weiterbildungskurse, ferner Unterrichtseinheiten zur Auswahl mit kurzen Filmen, mit einschlägigen Dias und Tonbandaufnahmen und ein großzügigeres Angebot von seiten der Ethnographischen Museen an Anleitungen zur Gruppenarbeit. Es hat sich zweifelsohne manches in dieser Richtung getan. Die Frage ist: Sollte nicht schon in der Basisausbildung der Ethnologen und Geographen die gegenseitige Ergänzbarkeit vermehrt betont werden? Der Trend zur Humangeographie einerseits und das rezente Interesse mancher Völkerkundler für ökologische Fragen sind ein günstiges Vorzeichen für eine intensivere Begegnung. Die ehrwürdigen Geographisch-Ethnographisch/Ethnologischen Gesellschaften von Zürich und Basel könnten auch heute wieder die Brücke zu erneuter aktiver Zusammenarbeit werden.

\section{Anmerkungen:}

1) WOLFGANG RUDOLF, Ethnologie: Zur Standortbestimmung einer Wissenschaft (Das wissenschaftliche Arbeitsbuch VIII/21) Tübingen, E. Huth, 1973: 49.

2) Siehe folgende Seite

3) Den Vertretern der einzelnen Institute, welche die Angaben zusammengestellt haben, sei hier gedankt. 